\title{
Capability-Based Business Model Transformation
}

\author{
Martin Henkel, Ilia Bider, and Erik Perjons \\ DSV, Stockholm University, Forum 100, 16440 Kista, Sweden \\ \{martinh, ilia, perjons\}@dsv.su.se
}

\begin{abstract}
Any organization in subject of changes in the environment, or having the desire to improve, needs to change their processes, personnel and their use of resources. Changes, may they be called for by external threats or opportunities or internal strengths or weaknesses, take their departure in an organizations existing capabilities. To support change, there is thus a fundamental need to understand and analyse an organizations capabilities in order to perform changes. In this paper we present an approach to support organizational change by the use of a capability based recursive analysis, and a set of improvement patterns. The recursive analysis is based on resource types, and capability sub-types. We illustrate the approach by using several examples taken from the industry.
\end{abstract}

Keywords: Dynamic Capability, Capability Engineering, Enterprise Modelling.

\section{Introduction}

Modern organizations work in ever changing environments. They constantly need to adopt their capabilities to meet new demands from customers and in order to increase their efficiency. Failure of an organization to adapt to radically changing environments can endeavour organizations profitability or even its existence [1]. There are no signs that the pace at which the organizations need to adapt to changes is slowing down. On the contrary, organizations that have a competitive advantage tend to keep that advantage for a shorter time [2]. Thus, to survive and grow in a dynamic environment with global competition for customers, capital and skilled workforce, a modern enterprise needs to quickly adapt its capabilities and business model to changes in the environment. If successful, organizations can use the opportunities these changes offer for launching new products and services.

Adapting to changes in the environment can be a complex task. First, an organization needs to know its current state. Secondly, there is a need to identify and understand the changes that need to be carried out.

There are several ways to describe an organizations current state. One established way is to describe an organization from a resource based view. Taking a resource based view [3] entails examining an organization's resources in the form of tangible and intangible assets under the control of the organization. These resources include the products an organization owns, its personnel skills, its processes, and the information it controls. An organization can, though, make use of its resources in 
different ways. Therefore, the concept of capability has been introduced. Capability can be defined as the ability of an organization to manage its resources to accomplish a task. The capabilities of an organization are thus tightly tied to the management of to its resources.

The resource based view has later been adopted to describe the necessity for organization to be dynamic, and thus develop skills to have "dynamic capabilities" [4]. The problem is, however, that little attention has been paid to providing organizations with a practical, easy-to use approach to analyse its existing capabilities, and to change them.

The long-term goal of our research is to provide a practical approach to model, and change an organization based on its existing capabilities. This paper presents an initial approach that achieves this, based on two main contributions, namely a modelling approach that allows the identification and description of organizational capabilities and patterns that facilitate change. We ground our approach in an industrial case, showing its initial viability.

The first contribution is a modelling approach that describes an organization as a recursive structure of capabilities, including the resources being used. The objective of having such a model is to help an enterprise to better understand its existing structure of capabilities so that it could be fully exploited and/or improved. Note that the creation of this form of model is not a trivial matter, as only the most visible processes usually catch attention of management and consultants. These easy-to-find processes represent only the tip of an iceberg of processes that exists in the enterprise, often in a half-documented, or in a totally undocumented form.

Our second contribution is a set of initial transformation patterns that allows an organization to find out possible new capabilities. These patterns rely on the previously defined model of capabilities. The approach presented in this paper fulfils one of the research topics as presented by the authors in [5]. In particular we introduce an approach tailored for identifying capabilities, and introduce new patterns for changing an organization.

This paper is structured according to the following. The next section sets this research in the context of related research. Section 3 gives and overview of the approach and defines its key concepts. Section 4 describes the first part of the approach, the recursive modelling of capabilities. Section 5 describes a set of patterns for discovering beneficial changes to the capabilities. Section 6 demonstrates the approach by applying it to an industrial case.

\section{Related Research}

Using capabilities as the foundation to describe organizations is not new, and can be found in the research domain of strategic management and enterprise architecture. Furthermore, recursive approaches to describe an organization, have been applied in the area of enterprise modelling. The research about these two concepts, capabilities and recursive approaches to describe an organization, are further described in this section. 
In strategic management the notion of dynamic capability can be used to describe the key properties that makes and organization withstand and make use of change. Analysis of existing companies has revealed differences in how successful companies manage change [6]. Even though the concept of capability has fluctuated in the area of strategic management, there is a tendency to associate it with resources and their allocation [7]. We use this interpretation of capability, that is, we view resources and their use as an integral part of capabilities. Frameworks such as Barneys VRIN [3] (Valuable, Rare, In-imitable, Non-sustainable) has been suggested as a way to find the resources that constitutes a company's competitive advantage. In contrast to the VRIN framework, our target is not to only elicit the resources that are company unique, we rather provide a tool for analysing all capabilities of an organisation.

In the strategic management area several authors are focusing on business transformations. These transformations can target finding a non-competitive area for products [8], or more general changes to the business model [9]. However these transformations, or patterns, only work on a high-level and thus run the risk of missing business opportunities buried in the lower levels of an organization. The recursive approach presented in this paper can be combined with the above mentioned approaches, since it adds the possibility to in detail analyse an organization.

In the area of enterprise modelling and architecture, the concept of capability has been used as a mean to develop dynamic IT solutions [10], and also to describe an organizations readiness for using enterprise architecture. This is most notable in the open group TOGAF framework [11], where capability frequently refers the architecture readiness of an organization. In general enterprise models has been used before as a step-wise way to analyse and change an organisation [12]. Our modelling approach is however particularly focusing on capabilities and is applying a recursive structure.

Analysis of enterprises based on the idea of a recursive structure has been done by several researchers and practitioners using the concept of fractal organisations, e.g., [13], [14]. In essence, fractals are a high-level abstract idea of a structure with a recurring (recursive) pattern repeating on all levels. Hoverstadt [13] uses the viable system model (VSM) to unfold the fractal structure of the enterprise via the system subsystems' relationships. Our long term goal is similar to Hoverstadt's: create an approach for modelling an enterprise as a multi-layered complex system. However, we use a different approach to enterprise modelling, instead of system subsystems relationships, we interleave capabilities and resources when building an enterprise model. Moreover, Sandkuhl and Kirikova [14] present the idea to find fractal structures in an enterprise model built when using a general modelling technique. The approach in [14] radically differs from that of ours. We have a hypothesis of a particular fractal structure to be found when analysing an enterprise, while [14] is trying to find any types of the fractal structures based on the generic characteristics of organizational fractals. 


\section{Overview of the Approach}

The approach presented is this paper is based on the idea that an organization's capabilities can be used as a foundation for describing and changing the organisation. Following a resource based view [3], we define a capability as the ability of an organization to manage its resources to accomplish a task. As pointed out in [7] an organization can only be said to exhibit a certain capability if it is able to repeatedly apply it. In essence this also means that the organization must have the resources and that the right capacity of those resource to perform the tasks. In this paper we build on this view of capabilities and use the notion of resource types (e.g. people, infrastructure), and tasks in the form of execution templates (e.g. a documented manufacturing process).

The approach is built upon two main steps. The first step consists of uncovering an organisations structure by recursively applying the concepts of capabilities and resources. This is aided by the use of a set of capability resource types and a set of capability sub-types. The second main step consists of transforming the organizational structure to construct a more viable structure. This second step is aided by the use of a set of simple transformation patterns. These two steps are described in detail below:

Step1, uncovering the organizational structure, is performed according to the following procedure. One starts with the visible part of the iceberg, so-called main capability. Here, as main we count capabilities that produce value for which some of the enterprise external stakeholders are ready to pay, e.g., customers of a private enterprise, or a local government paying for services provided to the public. Typical examples of main capabilities are product manufacturing, or service delivery (e.g., educational process at a university). When the main capabilities are identified, one proceeds "under water" following up resources that are needed to run the main capability. Each resource type requires a set of so-called supporting capabilities to have the corresponding resources in "working order" waiting to be deployed in the main capability. To supporting capabilities belong, for example, human resources (HR) (e.g., hiring or retiring members of staff) that ensure the enterprise having right people to be engaged in its main activities.

To convert the procedure above into a procedure that could be easily used in practice, we introduce:

- Capability resource types that aid in the identification of the resources that are a part of a particular capability. This is especially important as a starting point to unwind an organizations main capability.

- Capability sub-types that aid the exploration of supporting capabilities that are needed for each resource that are being part of a (main) capability.

Having these resource types and capability sub-types will help us to unveil the dynamic structure of an organisation starting from the main capability and going downwards via repeating pattern capability, its resources, sub-capabilities for each resource, resources for the sub-capability and so on. As the result we will get an indefinite tree consisting of the same type of elements. Such kind of structures is known in the scientific literature under the name of fractal structures [15]. As the 
result we will get an indefinite tree consisting of the same type of elements. Such kind of structures is known in the scientific literature under the name of fractal structures [15].

Step 2 aims at transforming the structure identified during step 1. Essentially the aim is to identify sub-capabilities that can be transformed into more customer oriented capabilities, thereby creating a new business model for the organization. A part of this transformation process is to examine if there need to be a change in the resources being used, and the needed capacity of the resources. The transformation can be done in several different ways, however in this paper we focus on supporting an initial transformation by using transformation patterns:

- A capability transformation pattern describes a modification of a capability structure (see step 1) into a new structure.

Based on the description above, the goal of this paper is to introduce the resource types, capability sub-types and transformation patterns, and show how to use them in practice to untangle and improve the dynamic structure of an organisation.

The research presented in the paper is done in the frame of the design science paradigm. The goal of such kind of research is finding and testing a generic solution, or artefact, for a class of practical problems [16]. The resource types, capability subtypes and transformation patterns and procedure of using them suggested in this paper constitute the design science artefact.

\section{$4 \quad$ Identifying Organisational Capabilities}

In the previous section we gave an overview of the approach, in this section we go into details about the first step - uncovering the organizational structure. We thus first introduce (Section 4.1) how to identify capabilities and their resources before going into details on how to find sub-capabilities (Section 4.1).

\subsection{Applying Capability Resources Types}

We consider as enterprise any organization where the operational activities of which are financed by external stakeholders. It can, for example, be a private company that gets money for its operational activities from the customers, a head office of an interest organization that gets money from the members, or a public office that gets money from the taxpaying citizens or inhabitants. We consider a main capability of an enterprise to be a capability that produces value to the enterprise's external stakeholders for which they are willing to pay. Note here that the concept of capability includes the resources being used to produce value. Our definition of main capability is thus not the same as the concept of core capability. Core capability refers to the inner sub-capabilities that are unique within a market segment and gives an enterprise competitive advantage [17]. For example, we do not consider as main capability neither sales nor marketing processes, nor product development processes in a product manufacturing company. 
To ensure that the enterprise can make use of its capabilities, it is essential that it got the necessary resources and that the capacity of the resources match the need. To describe the resources, is it practical to employ some form of classification scheme. An initial classification of resources coupled with enterprise capabilities is to divide resources into physical infrastructure resources, workforce in form of human capital, and organizational capital resources [3]. Organizational capital resources include the management plans and coordination to perform actions. In order to simplify the identification of resources we use this classification with the following additions: Firstly, in order to describe an enterprises relations to external stakeholders we introduce paying stakeholder (as described above), and partners. Moreover, to focus on the documented management procedures, we refer execution templates. We thus make use of the following resource types:

- Paying stakeholders. Examples: customers of a private enterprise, members of an interest organization, local or central government paying for services provided for the public.

- Workforce - people trained and qualified for employment. Examples: workers at the conveyor belt, physicians, researchers.

- Execution templates (ET). Plans of activities governing the use of resources, such as management policies and document methods. For example, for a production process in a manufacturing company, ET includes product design and design of a technological line to produce the product. For a software development company that provides customer-built software, ET could include a software methodology (project template) according to which their systems development is conducted.

- Partners. Partners provide the enterprise with resources needed for the organizational capabilities, e.g., electricity (power provider), money (banks or other type of investors), parts, etc. Partners get compensation for their products and services in form of payment, profit sharing, etc. Partners can be other enterprises or individuals, e.g., retired workers that can be hired in case there is temporal lack of skilled workforce to be engaged in a particular process instance.

- Infrastructure - equipment required for running the main task. Examples: production lines, raw materials, computers, communication lines, buildings, software systems etc.

The list of resources types can be extended, and made more fine granular. However, the above list makes a practical foundation for untangling an enterprise's structure. Below we give some additional clarification on the list of resources above.

- All resources are equally important, thus the order in which the resource types are listed is arbitrary.

- The resource types are mostly describing intangible resources. This can make them clash with the notion of resource as used in the world of finance [18]. Except the infrastructure, all resources listed above belong to the category of so-called intangible assets of the finance world.

- To be of use, each resource need to available in the right capacity. For example, hosting a niche website with few users may use a similar infrastructure as a popular website with high load, however the capacity of the infrastructure need to be vastly different. 
To identify the main capability of an enterprise according to the above description an analysts can ask the question "What are the capabilities that produces value to the enterprise's external stakeholders for which they are willing to pay?". The resource types can then be used to drive the exploration of the main capability. Figure 1 contains an example in graphical form. The figure contains the main capability "Production manufacturing" in an ellipse and its constituent resources using rectangles. The arrows from the oval indicates the dependency that the capability need the resources to function, the resource type of each resource is indicated as a label on the arrows. If desired, the needed capacity of the resources could be indicated on the arrows (e.g. that the capability needs 10 workers). Note that the example includes two resources of type "Execution template".

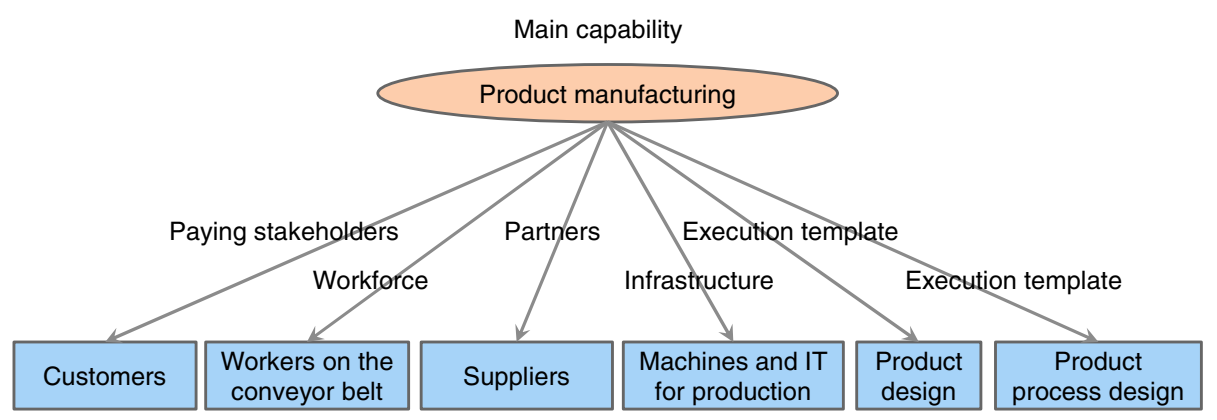

Fig. 1. An example of a main capability and its resources

As shown in the example, the resource types are used to detail the inner workings of a capability. The concept of capability is thus encompassing the resources and their correct configuration in the form of the needed capacities. To further explore an organization, we need to examine each resource in more detail.

\subsection{Capability Sub-types}

In the previous section we described how to identify main capabilities and their inherent resources. However each resource also needs to be managed to ensure that the overall capability is upheld. For example, an enterprise may need to work to promote their products in order to get new customers, or hire new workers if some workers leave. This handling of resources can be viewed as sub-capabilities. Indeed, some authors point out that this type of resource handling can be crucial for an enterprise and be an essential core capability of the enterprise [17].

To aid in the identification of sub-capabilities we make use of a simple acquire-maintain-retire resource lifecycle associated with each resource:

1. Acquire - capabilities that result in the enterprise acquiring a new resource of a given type. The essence of this capability depends on the type of resource, the type of the main capability in which it is used and the type of the enterprise. For a 
product-oriented enterprise, acquiring new customers (paying stakeholders) is done through its marketing and sales capabilities. Acquiring skilled work force is a task completed inside a recruiting process. Acquiring a new product design (an execution template) for a product-oriented enterprise is a task of new product and new technological process development.

2. Maintain - capabilities that help to keep existing resources in the right shape to be useful in the capability of a given type. For customers (paying stakeholder resource type), it could be the capability to perform Customer Relationship Management (CRM). For workforce, it could be training. For an execution template, it could be process or policy improvements. For technical infrastructure, it could be a utilized cloud service provided by a partner.

3. Retire - sub-capabilities that phase out resources that no longer can be used as part of the capability. For customers, it could be discontinuing serving a customer that is no longer profitable. For execution templates, it could be phasing out a product design that no longer satisfies the customer needs. For workforce, it could be actual retirement.

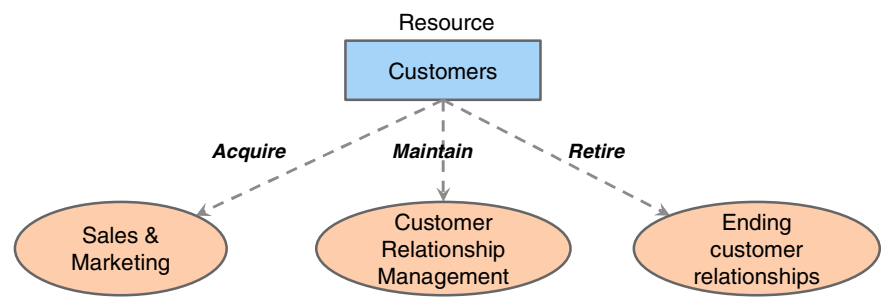

Fig. 2. An example of applying the sub-capability types

The sub-capability types can be graphically presented in the form of figure 2 . Figure 2 contains an example of a customer resource in a manufacturing company. Just as for figure 1, the resource is represented by a rectangle, and (sub-) capabilities by ovals. An arrow from the resource to a capability shows that the capability is aimed at managing the given resource. The label on the arrow shows the type of the capability in relation to its resource - acquire, maintain, or retire.

\section{$5 \quad$ Changing Organisational Capabilities}

The work with identifying capabilities lay the groundwork for proposing changes in the structure. The impetus for changing the structure of and enterprise can be both threats from competing enterprises and external business opportunities, such as a growing market segment. As pointed out by Chesbrough [19], experimentation is an essential part of changing the way an enterprise conducts its business. Moreover, Chesbrough argues that a suitable model of the enterprise can be used as a starting point for this experimentation. The presented structure of capabilities, resources and sub-capabilities can be used for this kind of experimentation. However, rather than having an ad-hoc approach for the experimentation itself we propose to use a set of 
generic capability transformation patterns. To illustrate their main use in this paper we here present two basic patterns that can be applied to the tree structure identified in the earlier step of the approach:

- Externalising a capability. This involves taking a capability that the enterprise has and market it toward its customers. For example, the well-known on-line book retailer Amazon has turned its internal IT platform into a service that customers can buy. In essence, what Amazon did was taking an internal sub-capability and turned it into one of its main capabilities (note that we defined a main capability to be the ones directly providing values to paying customers).

- Add value to a capability. While externalising a capability turns an existing capability into an organisation main capability, there is also the possibility to extend existing capabilities, or embed them into new main capabilities. For example, the ERP system provider Microsoft has plans to provide their ERP system Dynamic AX as a service hosted on Microsoft Azure based data centres. This move builds upon Microsoft capability to create ERP systems, combined with their capability to host cloud platforms.

To leverage the capability structure that was defined earlier, the above patterns can be applied to the identified capabilities. In practical terms this involves finding possible candidates for externalisation (the first pattern) by, for each capability, asking "Can this capability deliver values directly to customers?". The use of the added value pattern (the second pattern) can be assessed by in a similar manner asking "Can we combine this capability with other capabilities in order to provide value to our customers?". Examining the existing structure using the patterns provide a structured form of experimentation with an enterprises capabilities.

\subsection{Recursively Analysing the Capabilities}

By combining the described resource types with the capability sub-types it is possible to step-by step discover the organizational structure in form of capabilities. Potentially the resulting tree will grow in width and in breadth indefinitely. As an enterprise has a limited size, there should be some mechanisms that limit this growth and, eventually, stops it. We see several points at which the growth of the tree will stop:

- Outsourcing. Some capabilities, e.g., maintenance of infrastructure, can be outsourced to a partner. In this case, the partner will be responsible for a resource in the tree. If desired, the analysis could continue, to also cover the inner capabilities of the partner organisation. This could for example be useful if there is a doubt about the partners capacity.

- Reusing capabilities. A capability on an upper level of the tree can be employed as a supporting capability on the lower level, which terminates the growth from the corresponding node. For example, the capability to provide customer support for products could also be used internally within an organization. 
The above points can be combined with other ways of limiting the scope of the analysis, for example by scoping the analysis to a certain domain within the organization.

\section{Applying the Approach}

To illustrate the approach presented in this paper we have applied it to describe the changes a software development company went through. One of the main authors previously worked in this software company, and thus has experience with its way of working and the organisational changes that was applied. The changes described here took several years to perform, so we thus simplify the description.

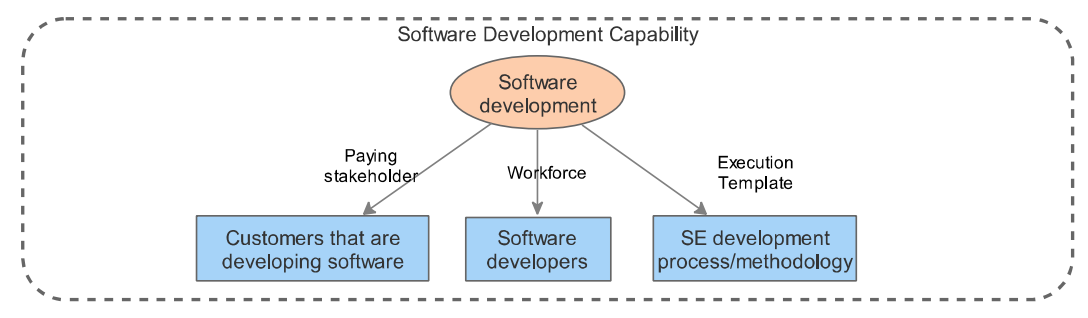

Fig. 3. Initial capability and its resources

Initially the company was a traditional software consulting company, working in several domains such as healthcare and financials. Its main capability (the main capability being defined as the one providing value to paying customers) was that of software development. Thus, the company worked as consultant for other organisation that developed custom made software. This capability consisted of the use of a set of resources as depicted in Figure 3. Most notably the organisation employed an execution template in form of a software development methodology (see figure 3 ).

As the time passed the company lost one of its main customers, leaving the company with several software developers without a project. To sustain, the company was thus in need of re-organising its capabilities. The re-organisation that was done can best be described using the added-value capability pattern described in the previous section. This means that the company looked for ways to build upon its existing capabilities by extending them. The extension in this case was to use the software development capability to develop a capability to build and distribute software packages. Figure 4 illustrates the new model describing this change.

The transformation that was made naturally also affected the use of the company's resources. As depicted in figure 4, the company could keep on utilising the main resources for software development, with the addition of procedures (execution templates) for generating new ideas for software products and their features. In addition to this the need for new capabilities to maintain and retire software products was added (see figure 4). The new main capability, software package distribution, also needed product delivery procedures (an execution template resource, see figure 4). 


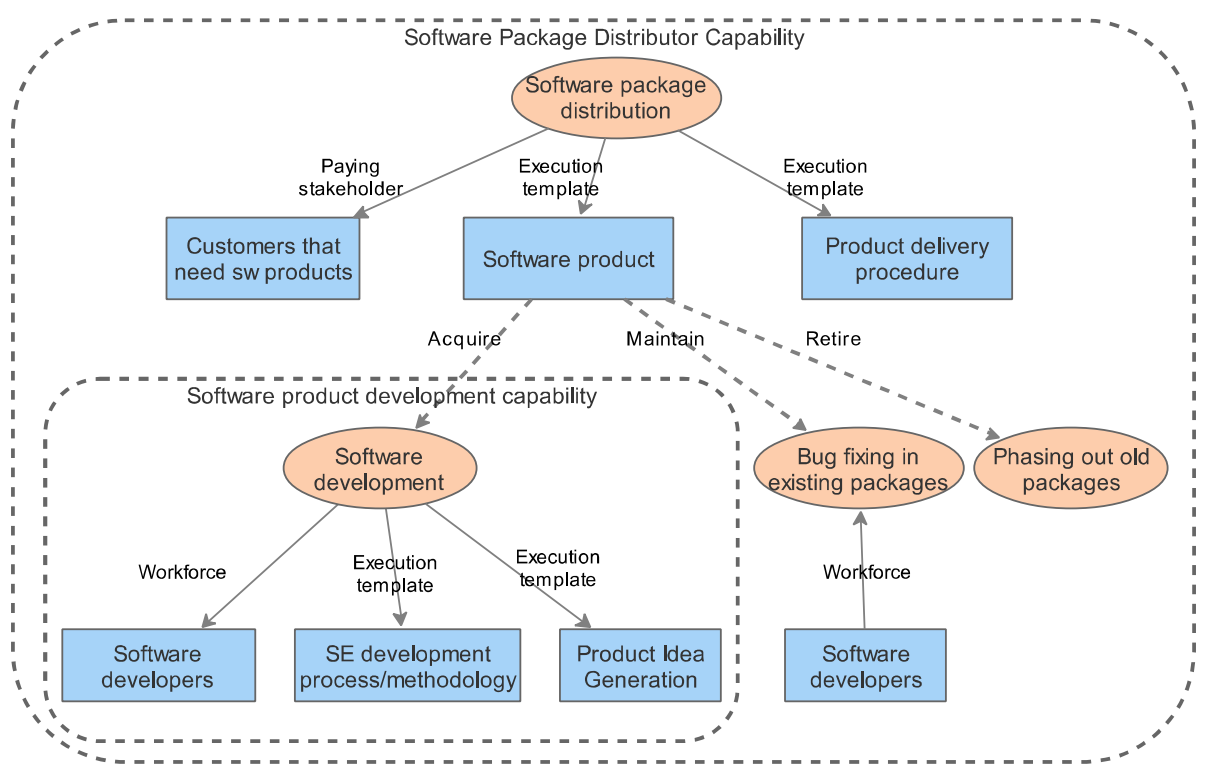

Fig. 4. New capability, embedding the existing capability

\section{Conclusion}

In this paper we presented an approach that help organisations to discover their inner structure and evolve it. The approach is based on that an organisation can be viewed as consisting of a multitude of capabilities, and that each capability requires specific resources of the correct capacity. To aid a recursive analysis of an organisation's capabilities, we introduce a set of capability resource types and a set of capability subtypes. Moreover, we presented two initial patterns that can help organisations to reorganise their capabilities in the event of threats or opportunities. The approach was illustrated by using an industrial case.

We see two possible ways for the future validation of the approach; discerning the utility by letting business consultant apply it to a real case and using historicalempirical validation in which the approach are used to explain existing organisational transformation.

Acknowledgements. This work has been partially supported by the EU-FP7 funded project no: 611351 CaaS - Capability as a Service in Digital Enterprises.

\section{References}

1. Audia, P.G., Locke, E.A., Smith, K.G.: The paradox of success: An archival and a laboratory study of strategic persistence following radical environmental change. Academy of Management Journal 43(5), 837-853 (2000)

2. Wiggins, R.R., Ruefli, T.W.: Schumpeter's ghost: Is hypercompetition making the best of times shorter? Strategic Management Journal 26(10), 887-911 (2005) 
3. Barney, J.: Firm resources and sustained competitive advantage. Journal of Management 17(1), 99-120 (1991)

4. Teece, D.J.: Explicating dynamic capabilities: the nature and microfoundations of (sustainable) enterprise performance. Strategic Management Journal 28(13), 1319-1350 (2007)

5. Bider, I., Perjons, E., Elias, M.: Untangling the Dynamic Structure of an Enterprise by Applying a Fractal Approach to Business Processes. In: Sandkuhl, K., Seigerroth, U., Stirna, J. (eds.) PoEM 2012. LNBIP, vol. 134, pp. 61-75. Springer, Heidelberg (2012)

6. Miller, D., Hope, Q., Eisenstat, R., Foote, N., Galbraith, J.: The problem of solutions: balancing clients and capabilities. Business Horizons 45(2), 3-12 (2002)

7. Schreyögg, G., Kliesch, E.M.: How dynamic can organizational capabilities be? Towards a dual-process model of capability dynamization. Strategic Management Journal 28(9), 913-933 (2007)

8. Kim, W.C., Mauborgne, R.: Blue ocean strategy: How to create uncontested market space and make competition irrelevant. Harvard Business Press (2005)

9. Osterwalder, A., Pigneur, Y.: Business model generation: a handbook for visionaries, game changers, and challengers. John Wiley \& Sons (2010)

10. Stirna, J., Grabis, J., Henkel, M., Zdravkovic, J.: Capability Driven Development - An Approach to Support Evolving Organizations. In: Sandkuhl, K., Seigerroth, U., Stirna, J. (eds.) PoEM 2012. LNBIP, vol. 134, pp. 117-131. Springer, Heidelberg (2012)

11. Open Group Standard, TOGAF - Enterprise Architecture Methodology, Version 9.1 (2011), http : / / www . opengroup.org/togaf / (accessed March 01, 2014)

12. Henkel, M., Johannesson, P., Perjons, E.: An Approach for E-Service Design using Enterprise Models. International Journal of Information System Modeling and Design (IJISMD) 2(1), 1-23 (2011)

13. Hoverstadt, P.: The Fractal Oragnization: Creating Sustainable Oragnization with the Viable System Model. John Wiley \& Son (2008)

14. Sandkuhl, K., Kirikova, M.: Analysing enterprise models from a fractal organisation perspective - potentials and limitations. In: Johannesson, P., Krogstie, J., Opdahl, A.L. (eds.) PoEM 2011. LNBIP, vol. 92, pp. 193-207. Springer, Heidelberg (2011)

15. McQueen, P.: Physics and fractal structures. Journal of Statistical Physics 86, 1397-1398 (1997)

16. Bider, I., Johannesson, P., Perjons, E.: Design Science Research as Movement Between Individual and Generic Situation-Problem-Solution Spaces. In: Designing Organizational Systems, pp. 35-61. Springer, Heidelberg (2013)

17. Schoemaker, P.J.: How to link strategic vision to core capabilities. Sloan Management Review ( October 15, 1992)

18. Elliott, B., Elliott, J.: Financial Accounting and Reporting. Financial Times/Prentice Hall, London (2004)

19. Chesbrough, H.: Business model innovation: opportunities and barriers. Long Range Planning 43(2), 354-363 (2010) 